\title{
Processes of apoptosis and cell proliferation in uterine myomas originating from reproductive and perimenopausal women
}

\author{
Andrzej Plewka ${ }^{1}$, Danuta Plewka ${ }^{2}$, Paweł Madej ${ }^{3}$, Grażyna Nowaczyk ${ }^{4}$, \\ Karolina Sieron-Stoltny ${ }^{5}$, Beata Jakubiec-Bartnik ${ }^{1}$ \\ ${ }^{1}$ Department of Proteomics, Medical University of Silesia, Sosnowiec, Poland \\ ${ }^{2}$ Department of Histology, Medical University of Silesia, Katowice, Poland \\ ${ }^{3}$ Chair and Department of Gynecological Endocrinology, Medical University of Silesia, \\ Katowice, Poland \\ ${ }^{4}$ Department of Physiology, School of Health Care, Medical University of Silesia, Katowice, Poland \\ ${ }^{5}$ Department of Internal Medicine, Medical University of Silesia, Bytom, Poland
}

\begin{abstract}
We studied uterine myomas originating from females of reproductive age and from females of perimenopausal age. Uterine myomas represent benign tumors of the myometrium, and they develop frequently in women of reproductive age. The frequency of uterine myomas increases with age until women reach the menopause. The study included patients with a myomatous uterus, in the reproductive age or peri-menopausal age, independently evaluating small and large myomas. Myometrial alterations in their direct vicinity were evaluated independently of the myomas. The study included evaluation of immunolocalization of two index proteins which participate in myoma cells growth control: Ki-67 nuclear antigen and caspase 3. In women of reproductive age, both in small and large myomas, elevated immunostaining of Ki-67 was noted in parallel to low levels of caspase 3 staining, which indicated the ongoing process of proliferation. In women of peri-menopausal age with small or large myomas, no Ki-67 immunostaining was detected, while staining of caspase 3 manifested low levels. Proliferation in reproductive age women myomas is higher than in the peri-menopausal age. (Folia Histochemica et Cytobiologica 2011; Vol. 49, No. 3, pp. 398-404)
\end{abstract}

Key words: apoptosis, cell proliferation, uterine myomas, Ki-67, caspase 3

\section{Introduction}

Despite their frequent manifestation, little data is available regarding the growth and development of leiomyomas. Based on studies of the activity of glucose-6-phosphate dehydrogenase, and cytogenetic studies, all leiomyomas are thought to reflect clonal

Correspondence address: A. Plewka,

Department of Proteomics, Medical University of Silesia, Ostrogorska Str. 30, 41-200 Sosnowiec, Poland; tel.: (+ 48 32) 36414 30, fax: (+ 48 32) 36414 40;

e-mail: aplewka@sum.edu.pl expansion of a single myocyte [1]. Similarly to other tumors, probably numerous sensitive genetic targets are present, the lesions of which lead to dysregulation of a smooth muscle cell, resulting in its transformation to the common phenotype of a leiomyoma.

Despite the limited knowledge about the specific events leading to the development of leiomyomas, they have been shown to represent hormone-sensitive tumors [2-6]. Case descriptions and studies originating from the late 1970s demonstrated that leiomyomas appear at the start of the reproductive age; they grow under the influence of sex steroids and regress following the menopause [7]. Nevertheless, the roles 
of steroids and other growth factors in the initiation and growth of the tumors remain incompletely understood [8]. Most probably, the neoplastic transformation of smooth muscle cells to leiomyoma involves somatic mutations of genes playing roles in proliferation and apoptosis in a normal myometrium and in complex interactions between sex steroids and growth factors. However, the observations provided investigators with an insight into leiomyoma pathophysiology and represented the first step for understanding the growth potential of the ovarian steroid-dependent tumors $[2,3,9]$.

Extensive knowledge has accumulated on the role of hormones in the growth of leiomyomas. Particular attention has been devoted to the role of steroid sex hormones, estrogens and progesterone in the pathophysiology of leiomyomas and to the relationship between the hormones and known leiomyoma growth factors. Attempts have been made to indicate possible therapeutic advantages by taking into consideration other observations of leiomyoma growth under the influence of modulators of sex steroid receptors and their antagonists [2, 10-12].

Caspase 3, owing to its specificity and sensitivity, represents a reliable marker of cells undergoing the process of programmed cell death. Low levels of caspase 3 may indicate partial inhibition of apoptosis. Activation of the latter process takes place following cell stimulation to apoptotic death, which leads to cell destruction and apoptotic cell death [13-15].

Two index proteins, which participate in growth control in leiomyoma monoclonal cells, have been evaluated. Ki-67 and caspase 3 have been selected, which are helpful in evaluation of the course and characteristics of the uterine leiomyoma neoplastic processes. In order to implement the goal, we have aimed at demonstrating the importance of the evaluation of differences in expression of the selected proteins in tissues of the myometrium and of leiomyoma.

The chief aim of our study was to resolve the problem of how the proliferative potential and intensity of apoptotic processes differ between the myometrium of healthy females and females in whom leiomyomas have been demonstrated. Moreover, we compared the profiles between small and large myomas to determine how the age of the patient affects the proliferative and apoptotic processes in the disease.

Since most myomatous women remain under permanent supervision, and are not immediately subjected to surgery, any additional information is particularly important, as it would allow for non-invasive monitoring of this common disease.

\section{Material and methods}

Material. Recruitment of the patients and their clinical studies were conducted in the Chair and Department of Gynecological Oncology, Silesian Medical School in Katowice, while the studies related to protein localization were performed in the Department of Proteomics, Medical University of Silesia in Sosnowiec.

The studies included ten patients of reproductive age with no leiomyomas and ten patients of peri-menopausal age. The patients provided the lesion-free controls. Moreover, the study included 40 reproductive age patients with uterine leiomyomas (below 45 years of age, $\mathrm{FSH}<30 \mathrm{mIU} /$ $/ \mathrm{ml}$; samples were taken in follicular phase of menstrual cycle) and 40 peri-menopausal age patients with uterine leiomyomas (45-55 years of age, $\mathrm{FSH}>30 \mathrm{mIU} / \mathrm{ml})$. In each case, the group of 40 patients provided 20 preparations of small leiomyomas and 20 preparations of large leiomyomas.

The experimental material was sampled during surgery. It included a leiomyoma sample of $1 \times 0.5 \mathrm{~cm}$ and a sample of healthy tissue of a similar size, taken at a distance of no less than $4 \mathrm{~cm}$ from the margins of the myoma (the autologous control). The material was immediately placed in neutral buffered formalin.

The study protocol was approved by the Ethical Commission, Silesian Medical University in Katowice.

Characteristics of studied groups. Group $1-$ myometrium of menstruating women, in whom hysterectomy was performed for reasons other than uterine leiomyomas; the ten patients represented the lesion-free control; Group 2 - leiomyomas of $<3 \mathrm{~cm}$ in diameter, sampled from the uteri of menstruating women $(\mathrm{n}=20)$; Group $3-$ myometrium of menstruating women, sampled at a distance of no less than $4 \mathrm{~cm}$ from the margins of a small leiomyoma ( $\mathrm{n}=20$; autologous control); Group $4-$ leiomyomas of $>5 \mathrm{~cm}$ in diameter, sampled from the uteri of menstruating women $(\mathrm{n}=20)$; Group 5 - myometrium of menstruating women, sampled at a distance of no less than $4 \mathrm{~cm}$ from the margins of a large leiomyoma $(n=20$; autologous control); Group 6 - myometrium of non-menstruating women, in whom hysterectomy was performed for reasons other than uterine leiomyomas ( $\mathrm{n}=10$; lesion-free control); Group 7 - leiomyomas of $<3 \mathrm{~cm}$ in diameter, sampled from the uteri of non-menstruating women $(\mathrm{n}=20)$; Group 8 myometrium of non-menstruating women, sampled at a distance of no less than $4 \mathrm{~cm}$ from the margins of a large leiomyoma ( $n=20$; autologous control); Group 9- leiomyomas of $>5 \mathrm{~cm}$ in diameter, sampled from the uteri of non-menstruating women $(\mathrm{n}=20)$; Group $10-$ myometrium of non-menstruating women, sampled at a distance of no less than $4 \mathrm{~cm}$ from the margins of a large leiomyoma ( $n=20$, autologous control). 
Histology. The tissue samples were fixed in $4 \%(\mathrm{v} / \mathrm{v})$ solution of buffered formalin, for $24 \mathrm{~h}$, at $4^{\circ} \mathrm{C}$, dehydrated, cleared in xylenes and embedded in paraffin.

Sections $(5 \mu \mathrm{m})$ were mounted on silane-coated slides, de-waxed, and rehydrated. The review preparations were stained with hematoxylin and eosin (H-E).

Immunohistochemical studies. Detection of apoptosis undergoing cells took advantage of immunohistochemical tests, employing polyclonal rabbit antibodies to the active form of caspase 3 (Abcam, Cambridge, UK) and for detection of dividing cells mouse monoclonal antibodies were used against the nuclear Ki-67 antigen (Santa Cruz Biotechnology, Santa Cruz, CA, USA).

To unmask the antigens, sections were incubated in a water bath at a temperature of $95^{\circ} \mathrm{C}$ in Tris-EDTA solution, $\mathrm{pH} 9.0$ for $30 \mathrm{~min}$ and, then cooled at room temperature. Endogenous peroxidase activity was quenched using 1.5\% (v/v) solution $\mathrm{H}_{2} \mathrm{O}_{2}$ in methanol for $10 \mathrm{~min}$ and then slides were washed in PBS-Tween $20(0.05 \% \mathrm{v} / \mathrm{v})$ and blocked with $1 \%$ BSA for $60 \mathrm{~min}$.

After this, the slides were incubated with appropriate primary antibodies. The incubation was conducted overnight, at a temperature of $4^{\circ} \mathrm{C}$. The $\mathrm{ABC}$ technique was employed to visualize the bound primary antibodies. After washing in PBS-Tween-20, sections were incubated with biotinylated goat anti-rabbit or horse anti-mouse immunoglobulins (Vector Laboratories Inc.) for $30 \mathrm{~min}$, and were incubated with avidin-biotinylated peroxidase complex (Vector) for $30 \mathrm{~min}$. Diaminobenzidine (DAB) and $\mathrm{H}_{2} \mathrm{O}_{2}$ in PBS, $\mathrm{pH} 7.5$ were used as peroxidase substrates (Vector). Finally, the tissues were stained with Gill's hematoxylin, dehydrated, and cover-slipped. For negative controls, rabbit or mouse IgG were used.

Archives. Photographic documents were prepared using a light microscope with a photographic attachment. In order to accentuate the immune reactions, every reaction was documented by 10 photographs under $\times 200$ magnification $(\times 20$ objective and $\times 10$ ocular $)$ using a digital cameraequipped Nikon microscope.

Evaluation of immune reaction. In microscope preparations of every experimental group, labelled cells were scored, in which caspase 3 expression or cell nuclei with Ki-67 expression was demonstrated.

\section{Results}

\section{Ki-67 nuclear antigen}

In control uterine sections, originating from women with no diagnosed leiomyomas, either at reproductive age (Group 1) or peri-menopausal age (Group 6), immunolocalization of Ki-67 was observed only sporadically (Figures $1 \mathrm{~A}, \mathrm{~F}$ ). At magnification $\times 400$, only one positive cell nucleus could be seen per microscope field. The labelled cell nuclei represented mainly cell nuclei of cells in connective tissue stroma.

In the experimental group in which leiomyomas originated from the uteri of women of reproductive age, immunolocalization of $\mathrm{Ki}-67$ was noted in 12 preparations per 20 cases of small leiomyomas (Group 2; Figure 1B) and in four preparations per 20 cases of large leiomyomas (Group 4; Figure 1D).

In the group of young women, the number of labelled cells per microscope field was higher compared to the control. On average, three cell nuclei were positive in eight preparations, and four cell nuclei were positive in four preparations per 20 examined preparations (Figure 1B). It should be recorded that similar results were obtained in preparations originating from the vicinity of leiomyomas (Group 3; Figure 1C). The dividing cells included, mainly, endothelial cells and myocytes. In preparations representing large myomas, on average four positive cells nuclei were detected per microscope field (Figure 1D), while in their vicinity just three positive cell nuclei were detected per microscope field (autologous control; Group 5; Figure 1E). In large leiomyomas, dividing myocytes prevailed, whereas in their vicinity dividing endothelial cells prevailed.

In preparations representing leiomyomas originating from peri-menopausal women, practically no Ki-67 immunolocalization was observed (just individual positive cell nuclei per preparation), independently of the size of leiomyoma (Groups 7 and 9; Figures $1 \mathrm{G}, \mathrm{I})$. Also no positive reaction could be detected in preparations representing the vicinity of leiomyoma (autologous control; Groups 8 and 10; Figures 1H, J).

\section{Caspase 3}

In control groups, apoptosis could be detected in both age groups, but it was more frequent in the myometria of young women (Group 1), involving 3-5 cells per visual field in every preparation (Figure $2 \mathrm{~A}$ ). In cases of peri-menopausal women (Group 6), on average six cases demonstrated a single cell and four cases two cells per visual field, respectively (Figure $2 \mathrm{~F}$ ).

In the research groups in all cases including 20 cases of various size leiomyomas, originating from women of reproductive age, immunolocalization of caspase 3 was detected in eight cases of small leiomyomas (Group 2; Figure 2B) and in 16 cases of large leiomyomas (Group 4; Figure 2D). In every case, however, no more than 1-2 cells undergoing apoptosis could be noted per visual field. A similar degree 

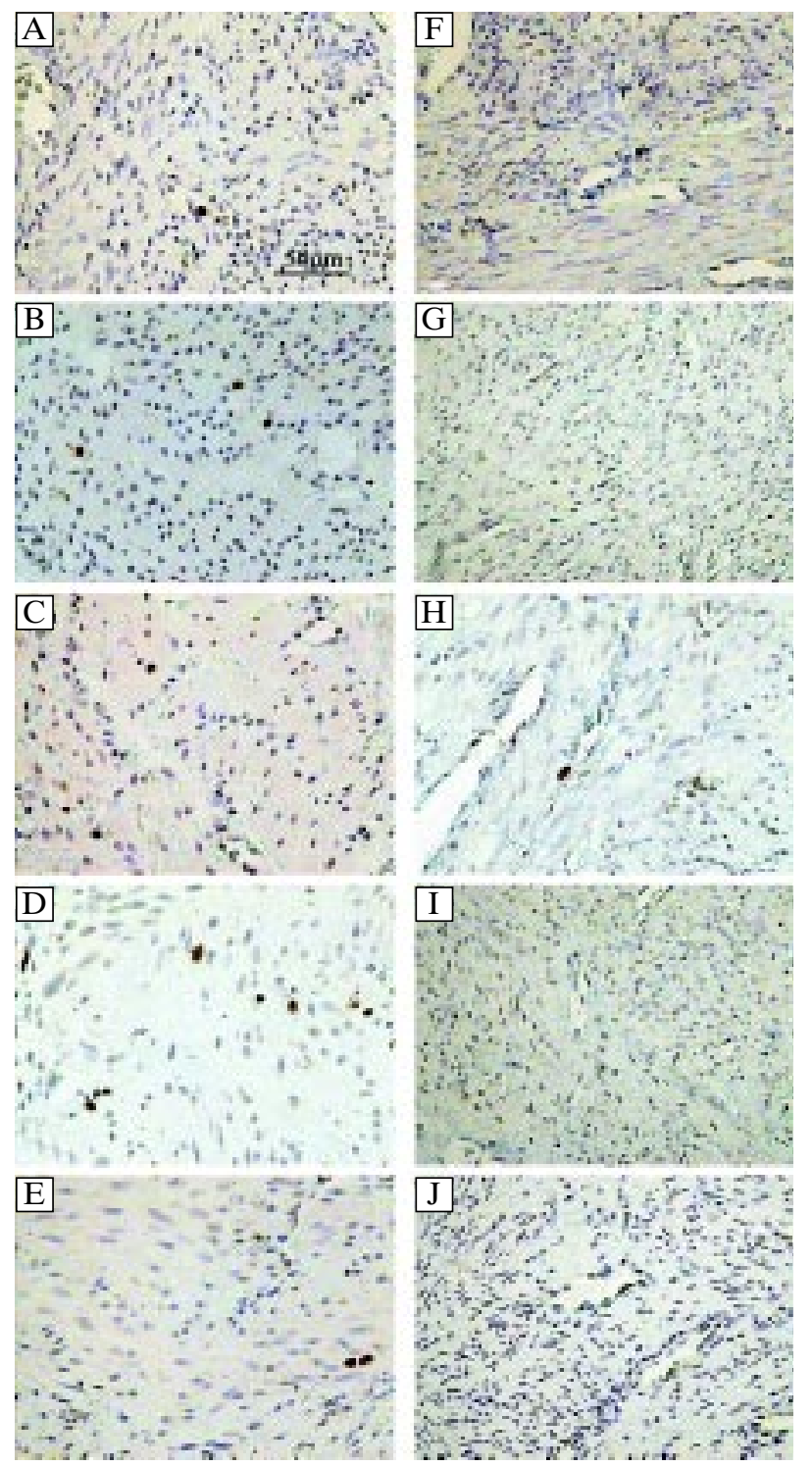

Figure 1. Immunohistochemical staining of uterine samples from women of reproductive $(\mathbf{A}-\mathbf{E})$ and perimenopausal (F-J) age with mouse anti-Ki-67 monoclonal antibodies. A and $\mathbf{F}$ - healthy myometrium; $\mathbf{B}$ and $\mathbf{G}-$ small myomas; $\mathbf{C}$ and $\mathbf{H}$ - periphery of small myomas; $\mathbf{D}$ and $\mathbf{I}$ - large myomas; $\mathbf{E}$ and $\mathbf{J}$ - periphery of large myomas

of immunological staining was also detected in the vicinity of the leiomyomas (autologous control; Groups 3 and 5; Figures 2C, E). It should be stressed that the apoptosis was not accompanied by proliferation: no immunolocalization of Ki-67 was detected in leiomyomas manifesting apoptosis.

In the group of uterine leiomyomas originating from peri-menopausal women, immunolocalization of caspase 3 was noted only in cases of small leiomyomas (Group 7; Figure 2G) and in their vicinity (autologous control; Group 8; Figure 2H). Expression of the caspase
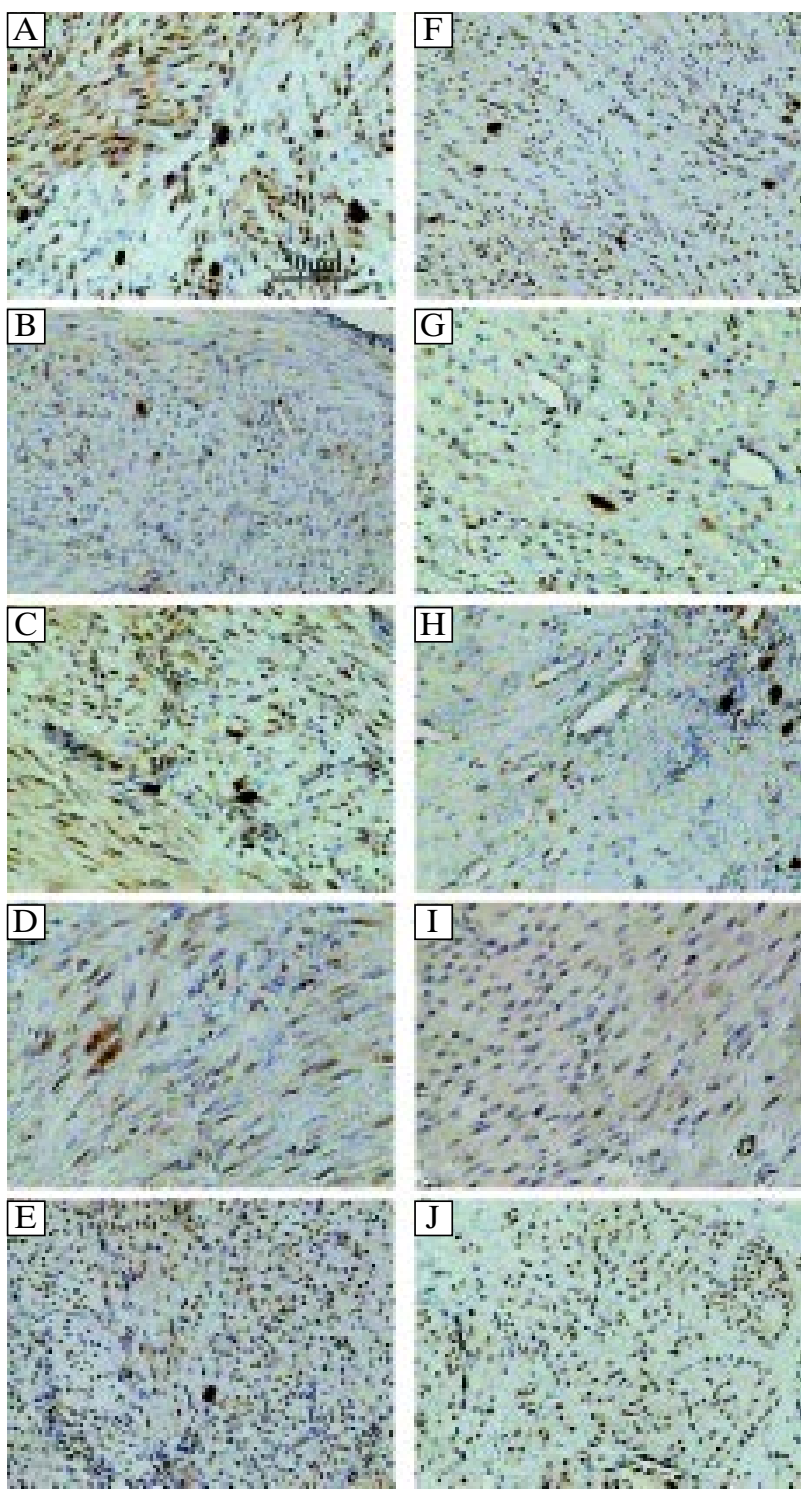

Figure 2. Immunohistochemical staining of uterine samples from women of reproductive (A-E) and perimenopausal (F-J) age with rabbit anti-caspase-3 polyclonal antibodies. A and $\mathbf{F}$ - healthy myometrium; $\mathbf{B}$ and $\mathbf{G}-$ small myomas; $\mathbf{C}$ and $\mathbf{H}$ - periphery of small myomas; $\mathbf{D}$ and $\mathbf{I}$ - large myomas; $\mathbf{E}$ and $\mathbf{J}$ - periphery of large myomas

ranged from one to two cells per visual field in cases of preparations representing the vicinity of leiomyoma, and at most a single cell in the leiomyoma tissue.

In summary, if they are manifested, proliferation processes (Ki-67 expression) can be noted only in myomas of women of reproductive age while apoptosis (active caspase 3 expression) does not develop in large myomas of women of perimenopausal age. Myometrium sampled close to a myoma manifests levels of proliferation and apoptosis similar to those seen in myoma tissue. 


\section{Discussion}

Among the evaluated indices related to links between apoptosis and cell proliferation, no immunolocalization of Ki-67 was detected in the control. This has been the case in both cases of samples originating from patients of reproductive age and postmenopausal women.

In the same samples, a well-marked activity of caspase 3 has been documented. Its level has been found to slightly vary depending on the age of the women: in the older group, the reaction for caspase 3 proved to be more intense. The pattern of immunocytochemical reactions changed in the myometrial samples obtained from women in whom leiomyomata had been detected. In all cases, a weak or a very weak immunolocalization of the two examined proteins was found. The alterations became even more accentuated: the activity of caspase 3 practically disappeared in leiomyomas, independently of the age group or leiomyoma size. Nevertheless, in the groups immunolocalization of $\mathrm{Ki}-67$ has been noted to change as a function of age. In leiomyomas originating from young women, activity of Ki- 67 has been detected even if its level has been low and has shown no relationship to the size of leiomyomas. The index of cell proliferation has been, however, absent in samples originating from post-menopausal women.

The absence of Ki-67 antigen points to the absence of growth signals. On the other hand, high immunostaining of caspase 3 in young women indicates the presence of apoptosis. In this case this reflects a physiological process, linked to phases of the menstrual cycle. In older women, expression of caspase 3 has been less pronounced since body senescence is accompanied by a decreased ability to induce apoptosis [16]. The decreased propensity to programmed cell death may reflect low levels of p53 and a high level of anti-apoptotic Bcl-2. Activity of caspase 3 cannot be detected before apoptosis; it grows in the course of apoptosis, beginning at its early stage, and it disappears again at later stages of apoptosis [13].

The results point to inhibition of apoptosis in leiomyomas obtained from women, whether they are in the reproductive period or not. However, the data on $\mathrm{Ki}-67$ suggests that proliferative processes take place only in young women. In such cases the size of the leiomyoma is irrelevant. Nevertheless, such argumentation must be very cautious and results related to the problem must be accompanied by an appropriate comment.

Regrettably, it should also be borne in mind that the proliferation-associated antigen manifests also its drawbacks: the amount of Ki-67 abruptly decreases following termination of mitosis. The protein is prac- tically undetectable in cells at $\mathrm{G}_{0}$ phase of cell cycle, which is linked to its half-life. Ki-67 protein cannot be detected in cells which remain out of the active cell cycle. For this reason, Ki-67 is thought to represent a reliable index of cell proliferation [17]. Nevertheless, certain problems have been noted related to the use of Ki-67 antigen as a prognostic index. The first of them involves the fact that the level of Ki-67 antigen expression is affected by the accessibility of nutrients for the cell. In cells devoid of such nutrients, no Ki-67 antigen presence can be detected. Leiomyoma cells, among others, are such cells. For example, examination of a tissue sample taken from the middle of a large leiomyoma with necrotic lesions may provide a falsely low level of the examined Ki-67 antigen. In turn, most tumors consist of a differentiated cell population, which may differ in expression levels of various proteins, including Ki-67. For example, two different samples isolated from the same tumor were examined for the presence of Ki-67 antigen and two clearly distinct values were obtained of proliferation index based on the protein.

Some authors have expressed reservations regarding the use of Ki-67 antigen as a cell proliferation index. Van Oijen et al. [18] and Ioachim [19] demonstrated that the protein can also be detected in cells in which the cell cycle was blocked. Already such information indicates that cells with Ki-67 antigen expression do not always remain in the active cell cycle. Reservations remain as to the use of Ki-67 antigen as an index of cell proliferation since most tumors consist of a differentiated cell population, which may differ in levels of expression of various proteins, including Ki-67 antigen. Occasionally, the number of proliferating cells estimated on the basis of Ki-67 antigen presence can be overestimated. A situation is also possible in which proliferating cells yield a negative result of the analysis. Analysis of MCM protein expression represents a more sensitive marker, markedly better than the currently employed conventional proliferation indices (Ki-67, PCNA) [4]. The studies demonstrated that application of antibodies directed against MCM proteins is more advantageous since, using such antibodies, higher numbers of cells were identified as present in the active cell cycle than those documented using PCNA or Ki-67. Further studies and analysis will be required to clarify whether or not such reservations also pertain to leiomyomas.

Cells of leiomyomas, compared to the surrounding smooth muscle cells, contain a much higher density of estrogen receptors. Estrogens synthesized in leiomyoma cells boost proliferation, mainly due to stimulation of aromatase activity, the expression of 
which is much higher in leiomyoma cells than in the tumor-surrounding smooth muscles. The presence of Ki-67 antigen outside the leiomyoma may indicate continuing hyperplasia of the tumor [20-24].

The absence of Ki-67 antigen in leiomyomas of females at the peri-menopausal age suggests the inhibition of proliferation might have been induced by the absence of stimulation by ovarian estrogens. The estrogens localized in tumor cells originate from aromatization and act locally as a mitogenic factor, exerting a promotional effect until a certain moment, following which estrogen concentration becomes normalized and stable. The absence of Ki-67 antigen also allows another conclusion to be reached: the tumor remains in a condition of equilibrium, devoid of nutrients and of growth factors. This reflects the absence of blood vessels in the neighbourhood of descendant cells. The low level of caspase 3 expression points to the undergoing apoptotic process, probably resulting in a reduction in tumor mass [14].

Uterine leiomyomas are known to be the most frequent tumors of the female genital tract. Independently of the usual uterine leiomyomas or leiomyosarcomas, a spectrum of intermediate uterine smooth muscle tumors has been documented, with overlapping characters. It remains unclear whether, at the level of pathogenesis, the intermediate tumors reflect transition from one marginal situation of common benign leiomyomas to the other extreme of uterine leiosarcomas [25, 26].

The challenge following diagnosis of uterine leiomyoma involves prognostic qualification of the case. It should be kept in mind that histopathological and clinical traits of benign and malignant tumors do not necessarily correlate with each other. The morphological traits remain as valuable as their capacity to predict clinical outcome within the accepted range of reliability. In most cases, leiomyoma cases are easily categorised as benign or malignant tumors. In such cases, morphological traits allow the reliable prediction of clinical behaviour. In other cases, however, correlation of morphological patterns with clinical prognosis remains a significant challenge [27, 28].

Evaluation of basic leiomyoma traits with usual differentiation should incorporate the key prognostic markers, such as presence or absence of coagulative necrosis, of moderate or severe atypia and mitotic index. Only following completion of such a list can an attempt be undertaken to evaluate clinically significant categories of leiomyomas.

If the mitotic index remains below 5 cell divisions per 10 high magnification microscope fields, the tumor is categorized as leiomyoma. A typical leiomyoma is usually well demarcated and white. At the mi- croscopic level, tumor cells resemble the surrounding uterine muscle cells, except that they are more crowded and the large ones have larger cell nuclei.

Additional immunohistochemical techniques have been employed to evaluate cases of unreliable histology [29-31]. Immune staining for Ki-67 may be useful in distinguishing leiosarcomas and tumors of uncertain and unknown malignant potential (STUMP) or uterine leiomyomas [32-34]. This can be exemplified by Mayerhofer et al. [35] and Lee et al. [36], who demonstrated expression of Ki-67 in every other preparation with uterine leiomyosarcomas, but in none of the examined cases of STUMP. In numerous studies, cell cycle proteins other than Ki-67 have been examined. For example, expressions of cyclins (E and A) were clearly augmented in leiomyosarcomas compared to leiomyomas and STUMP. Similar observations have been conducted in the cases of p16 protein, progesterone receptor and metalloproteinase-2 [37-39]. These and other studies represent strong factors which allow prediction of prognosis.

At present, the decision as to how to categorize uterine tumors of smooth muscle cells, which on the one hand differ from the most evident uterine leiomyomas, and on the other hand from leiomyosarcomas, continues to depend on the fixed conventional criteria. Determination of tumor category should also reflect clinical criteria and not just routine procedures. A multi-stage approach is required for more accurate clinical prognosis. It encompasses linking basic clinical data, intraoperative and macroscopic observations, numerous morphological criteria and the results of immunohistochemical tests.

In our opinion, in the future, with the accumulation of cases with specific clinical outcomes and morphological traits, and under the influence of a growing amount of data on molecular indices, the prognosis for management of such tumors will become much more objective.

\section{References}

1. Pandis N, Heim S, Bardi G et al. Chromosome analysis of 96 uterine leiomyomas. Cancer Genet Cytogenet. 1991;55:11-18.

2. Bulun SE, Zeitoun KM, Takayama K, Sasano H. Estrogen biosynthesis in endometriosis: molecular basis and clinical relevance. J Mol Endocrinol. 2000;25:35-42.

3. Marsh EE, Bulun SE. Steroid hormones and leiomyomas. Obstet Gynecol Clin North Am. 2006;33:59-67.

4. Maruo T, Ohara N, Wang J, Matsuo H. Sex steroidal regulation of uterine leiomyoma growth and apoptosis. Hum Reprod Update. 2004;10:207-220.

5. Ishikawa H, Ishi K, Serna VA, Kakazu R, Bulun SE, Kurita T. Progesterone is essential for maintenance and growth of uterine leiomyoma. Endocrinology. 2010;151:2433-2442.

6. Yoshida S, Ohara N, Xu Q et al. Cell-type specific actions of progesterone receptor modulators in the regulation of uterine leiomyoma growth. Semin Reprod Med. 2010;28:260-273. 
7. Bulun SE, Zeitoun K, Sasano H, Simpson ER. Aromatase in aging women. Semin Reprod Endocrinol. 1999;17:349-358.

8. Shozu M, Sumitani H, Segawa T et al. Overexpression of aromatase $\mathrm{P} 450$ in leiomyoma tissue is driven primarily through promoter I.4 of the aromatase P450 gene (CYP19). J Clin Endocrinol Metab. 2002;87:2540-2548.

9. Flake GP, Andersen J, Dixon D. Etiology and pathogenesis of uterine leiomyomas: a review. Environ Health Perspect. 2003;111:1037-1054.

10. Shozu M. Murakami K, Inoue M. Aromatase and leiomyoma of the uterus. Semin Reprod Med. 2004;22:51-60.

11. Meinhardt U, Mullis PE. The aromatase cytochrome P-450 and its clinical impact. Horm Res. 2002;57:145-152.

12. Imir AG, Lin Z, Yin P et al. Aromatase expression in uterine leiomyomata is regulated primarily by proximal promoters I.3/II. J Clin Endocrinol Metab. 2007;92:1979-1982.

13. Sobańska E, Bar J, Harłozińska-Szmyrka A, Rozdolski K. Relations between the expression of p53 protein, growth fraction (Ki-67) and the presence of steroid hormone receptors in endometrial carcinomas. Adv Clin Exp Med. 2001; $10: 45-51$.

14. Skirnisdóttir I, Seidal T, Gerdin E, Sorbe B. The prognostic importance of $\mathrm{p} 53$, bcl-2, and bax in early stage epithelial ovarian carcinoma treated with adjuvant chemotherapy. Int J Gynecol Cancer. 2002;12:265-276.

15. Porebska I, Sobańska E, Kosacka M, Jankowska R. Apoptotic regulators: $\mathrm{P} 53$ and survivin expression in non-small cell lung cancer. Cancer Genomics Proteomics. 2010;7:331-335.

16. Mulayim N, Gucer F. Borderline smooth muscle tumors of the uterus. Obstet Gynecol Clin North Am. 2006;33:171-181.

17. Linden MD, el-Naggar AK, Nathanson SD, Jacobson G, Zarbo RJ. Lack of correlation between flow cytometric and immunohistologic proliferation measurements of tumors. Mod Pathol. 1996;9:682-689.

18. van Oijen MG, Medema RH, Slootweg PJ, Rijksen G. Positivity of the proliferation marker Ki-67 in noncycling cells. Am J Clin Pathol. 1998;110:24-31.

19. Ioachim E. Expression patterns of cyclins D1, E and cyclindependent kinase inhibitors p21waf1/cip1, p27kip1 in colorectal carcinoma: correlation with other cell cycle regulators (pRb, p53 and Ki-67 and PCNA) and clinicopathological features. Int J Clin Pract. 2008;62:1736-1743.

20. Pavlovich SV, Volkov NI, Burlev VA. Proliferative activity and level of steroid hormone receptors in the myometrium and myoma nodes in different phases of menstrual cycle. Bull Exp Biol Med. 2003;136:396-398.

21. Vu K, Greenspan DL, Wu TC, Zacur HA, Kurman RJ. Cellular proliferation, estrogen receptor, progesterone receptor, and bcl-2 expression in GnRH agonist-treated uterine leiomyomas. Hum Pathol. 1998;29:359-363.

22. Zasławski R, Surowiak P, Dziegiel P, Pretnik L, Zabel M. Analysis of the expression of estrogen and progesteron receptors, and of PCNA and Ki67 proliferation antigens, in uterine myomata cells in relation to the phase of the menstrual cycle. Med Sci Monit. 2001;7:908-913.

23. Baytur YB, Ozbilgin K, Cilaker S et al. A comparative study of the effect of raloxifene and gosereline on uterine leiomyoma volume changes and estrogen receptor, progesterone receptor, bcl-2 and p53 expression immunohistochemically in premenopausal women. Eur J Obstet Gynecol Reprod Biol. 2007;135:94-103.

24. Sen N, Demirkan NC, Colakoglu N, Duzcan SE. Are there any differences in the expression of hormonal receptors and proliferation markers between uterine and extrauterine leiomyomas? Int J Surg Pathol. 2008;16:43-47.

25. Kempson RL, Hendrickson MR. Smooth muscle, endometrial stromal, and mixed Müllerian tumors of the uterus. Mod Pathol. 2000;13:328-342.

26. Scurry J, Kerdemelidis P, Fortune D. Small atypical leiomyomas: report of two cases. Pathology. 2001;33:319-321.

27. Esteban JM, Allen WM, Schaerf RH. Benign metastasizing leiomyoma of the uterus: histologic and immunohistochemical characterization of primary and metastatic lesions. Arch Pathol Lab Med. 1999;123:960-962.

28. Tietze L, Günther K, Hörbe A et al. Benign metastasizing leiomyoma: a cytogenetically balanced but clonal disease. Hum Pathol. 2000;31:126-128.

29. Mittal K, Demopoulos RI. MIB-1 (Ki-67), p53, estrogen receptor, and progesterone receptor expression in uterine smooth muscle tumors. Hum Pathol. 2001;32:984-987.

30. Petrović D, Babić D, Forko JI, Martinac I. Expression of Ki-67, P53 and progesterone receptors in uterine smooth muscle tumors. Diagnostic value. Coll Antropol. 2010;34:93-97.

31. Sun X, Mittal K. MIB-1 (Ki-67), estrogen receptor, progesterone receptor, and $\mathrm{p} 53$ expression in atypical cells in uterine symplastic leiomyomas. Int J Gynecol Pathol. 2010;29: $51-54$.

32. Zhai YL, Kobayashi Y, Mori A et al. Expression of steroid receptors, Ki-67, and p53 in uterine leiomyosarcomas. Int J Gynecol Pathol. 1999;18:20-28.

33. Mittal KR, Chen F, Wei JJ et al. Molecular and immunohistochemical evidence for the origin of uterine leiomyosarcomas from associated leiomyoma and symplastic leiomyomalike areas. Mod Pathol. 2009;22:1303-1311.

34. Chen L, Yang B. Immunohistochemical analysis of p16, p53, and Ki-67 expression in uterine smooth muscle tumors. Int J Gynecol Pathol. 2008;27:326-332.

35. Mayerhofer K, Lozanov P, Bodner K, Bodner-Adler B, Kimberger O, Czerwenka K. Ki-67 expression in patients with uterine leiomyomas, uterine smooth muscle tumors of uncertain malignant potential (STUMP) and uterine leiomyosarcomas (LMS). Acta Obstet Gynecol Scand. 2004;83:10851088.

36. Lee $\mathrm{CH}$, Turbin DA, Sung YC et al. A panel of antibodies to determine site of origin and malignancy in smooth muscle tumors. Mod Pathol. 2009;22:1519-1531.

37. Bodner K, Bodner-Adler B, Kimberger O, Czerwenka K, Mayerhofer K. Estrogen and progesterone receptor expression in patients with uterine smooth muscle tumors. Fertil Steril. 2004;81:1062-1066.

38. Bodner-Adler B, Bodner K, Czerwenka K, Kimberger O, Leodolter S, Mayerhofer K. Expression of p16 protein in patients with uterine smooth muscle tumors: an immunohistochemical analysis. Gynecol Oncol. 2005;96:62-66.

39. Comunoğlu NU, Durak H, Comunoğlu C et al. Expression of cyclooxygenase-2, c-kit, progesterone and estrogen receptors in uterine smooth muscle tumors: differential diagnosis. APMIS. 2007;115:726-735. 